\title{
Ensaio biomecânico após retirada de parafusos canulados do fêmur proximal (análise in vitro)*
}

\section{Biomechanical Test after Hip Cannulated Screw Removal (in vitro Analysis)}

\author{
Anderson Freitas ${ }^{10}$ Lucas S. Ramos ${ }^{2}$ Érgon LAB Dantas ${ }^{2}$ Vincenzo Giordano Neto ${ }^{3}$ \\ Patrick F. Godinho ${ }^{1}$ Antônio C. Shimano ${ }^{4}$
}

${ }^{1}$ Instituto de Pesquisa e Ensino do Hospital Ortopédico e Medicina Especializada (IPE-HOME-DF), Brasília, DF, Brasil

2 Serviço de Ortopedia e Traumatologia, Hospital Regional do Gama (HRG-DF), Brasília, DF, Brasil

${ }^{3}$ Serviço de Ortopedia e Traumatologia, Hospital Municipal Miguel Couto (HMMC-RJ), Rio de Janeiro, RJ, Brasil

${ }^{4}$ Departamento de Biomecânica, Medicina e Reabilitação do Aparelho Locomotor, Faculdade de Medicina de Ribeirão Preto, Universidade de São Paulo (USP-RP), Riberião Preto, SP, Brasil

\begin{abstract}
Endereço para correspondência Anderson Freitas, PhD, Instituto de Pesquisa e Ensino do Hospital Ortopédico e Medicina Especializada (IPE-HOME-DF), Brasil SHCS 613 Conjunto C, Brasília, DF, Brasília, 70200-730, Brasil (e-mail: andfreitas28@gmail.com).
\end{abstract}

Rev Bras Ortop 2019;54:416-421.

\section{Resumo}

\section{Palavras-chave}

- fraturas do quadril

- fraturas do fêmur

- osteoporose

- polimetilmetacrilato

Objetivo Avaliar, por meio de ensaio biomecânico, a resistência e a energia necessária para ocorrência de fratura do fêmur proximal em osso sintético após retirada de parafusos canulados em forma de triângulo invertido e comparar os resultados obtidos com técnica de reforço utilizando polimetilmetacrilato (PMMA).

Métodos Foram utilizados 20 ossos sintéticos: 10 unidades para o grupo controle; 50 grupo teste sem reforço, sem preenchimento após a retirada dos parafusos canulados, e 5 o para grupo teste com reforço com PMMA. A análise biomecânica foi realizada simulando queda sobre o grande trocânter utilizando máquina servo-hidráulica.

Resultados Todos os corpos de prova dos grupos controle e sem cimento apresentaram fratura baso-cervical. No grupo teste com preechimento, três corpos de prova apresentaram fratura baso-cervical, enquanto que dois deles apresentaram fratura na parte próxima ao ponto de fixação no dispositivo (região diafisária do fêmur), sendo um deles associado a fratura do colo femoral. Foi utilizada uma média de $8.2 \mathrm{ml}$ de polimetilmetacrilato no preenchimento dos três pertuitos do grupo com preenchimento. Segundo a análise de variância (ANOVA, na sigla em inglês) para um fator e o teste de comparações múltiplas de Tukey, ao nível de 5\%, o grupo com cimento apresentou diferença significativa em relação aos outros grupos em todos os parâmetros.
\end{abstract}

\footnotetext{
Trabalho feito no Serviço de Ortopedia e Traumatologia, Hospital Regional do Gama, Brasilia, DF e pelo Instituto de Pesquisa e Ensino do Hospital Ortopédico e Medicina Especializada (IPE-HOME), Brasília, DF, Brasil.

(D) Anderson Freitas's ORCID is https://orcid.org/0000-0001-6555-8193.
}

recebido

01 de Março de 2018

aceito

03 de Setembro de 2018
DOI https://doi.org/

10.1055/s-0039-1693046. ISSN $0102-3616$
Copyright $\odot 2019$ by Sociedade Brasileira License terms de Ortopedia e Traumatologia. Published by Thieme Revnter Publicações Ltda, Rio de Janeiro, Brazil 


\begin{abstract}
Keywords

- hip fractures

- femoral fractures

- osteoporosis

- polymethylmethacrylate

Objective To evaluate, through biomechanical test, the resistance and energy required for the occurrence of proximal femoral fracture in synthetic bones after removing cannulated screws in the inverted triangle shape. Furthermore, comparing the results found with the reinforcement technique by means of bone cement - polymethylmethacrylate (PMMA).

Methods Twenty synthetic bones were used: 10 units for the control group (GC), 5 units for the test group without reinforcement (GTS), and 5 units for test group using reinforcement technique with PMMA (GTC). The biomechanical analysis was performed simulated a fall on the large trochanter using a servo-hydraulic machine.

Results All GTS and GC specimens had a baso-cervical fracture. In GTC, three specimens presented a baso-cervical fracture while two of them presented it in the part near the fixation point in the device (diaphyseal region of the femur), one of them being associated with fracture of the femoral neck. A mean of $8.2 \mathrm{ml}$ of PMMA was used in filling the 3 screws hole of the GTC group. According to the one-way analysis of variance (ANOVA) and the Tukey multiple comparisons test at the $5 \%$ level, the GTC group presented a significant difference in relation to the other groups in all parameters: maximal load $(p=0.001)$ and energy until fracture $(p=0.0001)$.

Conclusion The simple removal of the cannulated screws did not present a significant reduction from the maximum load and energy for the occurrence of fracture, but the proximal femoral reinforcement with PMMA significantly increased these parameters, causing changes in the fracture pattern.
\end{abstract}

Conclusão A simples retirada dos parafusos canulados não apresentou redução significativa da carga máxima e da energia necessárias para a ocorrência de fratura; porém, o reforço do fêmur proximal com polimetilmetacrilato aumentou significativamente esses parâmetros, causando mudanças no padrão fraturário.

\section{Introdução}

A osteoporose é um problema de saúde pública para a população mundial, sendo essa doença de maior prevalência na população feminina idosa. A osteoporose é caracterizada pela redução da densidade mineral óssea, e, com isso, a diminuição da resistência mecânica deste tecido tem como principal fator de impacto sócio econômico a ocorrência de fraturas por trauma de baixa energia. Destas, a fratura do extremo proximal do fêmur (EPF) é a que possui o maior índice de morbi-mortalidade. ${ }^{1,2}$

O objetivo do tratamento cirúrgico dessas fraturas é dar condições à retomada das atividades habituais do paciente $o$ mais rápido possível, realizando a fixação da fratura por meio de hastes de fêmur proximal(HFP), parafusos canulados (PCs)e/ou placa tubo deslizante (DHS), e ainda a substituição articular por artroplastia do quadril a fim de diminuir a possibilidade de complicações clínicas devido à imobilidade do paciente, métodos estes já amplamente utilizados em nosso meio. ${ }^{3}$

As indicações de retirada das sínteses, utilizadas para o tratamento das fraturas do EPF, ocorrem por dor persistente nas regiões glútea e da coxa, que pode ser causada pela proeminência do material de síntese, comum no uso dos PCs pela sua habitual migração, ${ }^{4}$ seja ainda por falha do implante ou por infecção. Após a consolidação da fratura do fêmur proximal, a remoção de implantes pode causar complicações, tais como possíveis fraturas do colo femoral ou da região intertrocantérica, principalmente em paciente com baixa qualidade óssea. ${ }^{2,5,6}$

Com isso, descrever os resultados por meio de um ensaio estático de flexão, simulando queda sobre o trocânter em fêmures sintéticos, após a retirada de três PCs em forma de triângulo invertido, com a presença e a ausência de uma técnica de reforço, pode propiciar resultados que determinem o desenvolvimento de ensaios clínicos a fim de apresentar um maior cuidado nas indicações de retirada deste modelo de síntese.

\section{Material e Métodos}

Com o objetivo de avaliar a resistência e a energia necessárias para a ocorrência de fratura do fêmur proximal em osso sintético, após a retirada de PCs em forma de triângulo invertido, e comparar os resultados obtidos com técnica de reforço utilizando polimetilmetacrilato (PMMA), foram utilizados 20 espécimes de fabricação nacional, modelo c1010 (Nacional ossos, Jaú, SP, Brasil), fabricado em poliuretana esponjoso e cortical com 10 PCF (pounds per cubic foot, medida internacional de densidade) com canal medular de $12 \mathrm{~mm}$, de 
mesmo lote e modelo divididos em 3 grupos, um grupo controle (GC), com 10 fêmures; grupo teste sem reforço (GTS), com 5 fêmures; e grupo teste com reforço (GTC), com 5 fêmures.

O GC foi constituído por fêmures sintéticos com sua integridade externa e interna intactas. Nos grupos GTS e GTC, os fêmures sintéticos, sem a realização de fraturas prévias, foram submetidos à introdução de fio guia no formato de triângulo invertido equidistantes entre si, sendo o mais inferior introduzido na mesma linha do pequeno trocanter, com auxílio de radioscopia em todos os espécimes. Tais fios foram introduzidos até a distância de $5 \mathrm{~mm}$ da superfície da cabeça femoral. A medida desses fios foi realizada com medidor padrão do fabricante nacional (Ortosintese Indústria e Comércio Ltda., São Paulo, SP, Brasil), determinando-se o comprimento dos parafusos separadamente. Os pertuitos foram realizados utilizando uma broca canulada própria do fabricante para a utilização de parafusos canulados de 7,5 mm e de comprimento previamente determinado pela medida adquirida.

No grupo GTS, o ensaio biomecânico foi realizado logo após a retirada dos implantes, sem utilização de qualquer técnica de reforço, no grupo GTC. Após a retirada dos implantes, os modelos sintéticos foram submetidos a uma técnica de reforço com o uso de cimento ósseo tipo PMMA (Biomecânica Indústria e Comércio de produtos Ortopédicos, Jaú, SP, Brasil) de viscosidade normal, preenchendo o pertuito dos parafusos canulados, que foi introduzido de forma anterógrada, com auxílio de uma seringa de $20 \mathrm{ml}$, e por meio desta foi calculado o volume utilizado de PMMA (-Fig. 1). Uma vez que alguns espécimes tiveram um preenchimento inadvertido de parte do canal femoral, com a ausência de PMMA na cabeça femoral, determinamos uma condição de inclusão: o preenchimento de todo o comprimento do colo femoral (podendo a cabeça estar sem preenchimento em somente um dos pertuitos) e preenchimento inadvertido da medular menor que $5 \mathrm{~cm}$ (-Fig. 1). Todas as amostras foram submetidas a ensaios estáticos de flexão, utilizando a máquina servo-hidráulica do modelo MTS 810-FlexTest 40 (MTS Landmark Testing Solutions, Eden Prairie, MN, EUA) com capacidade de 100 kN.

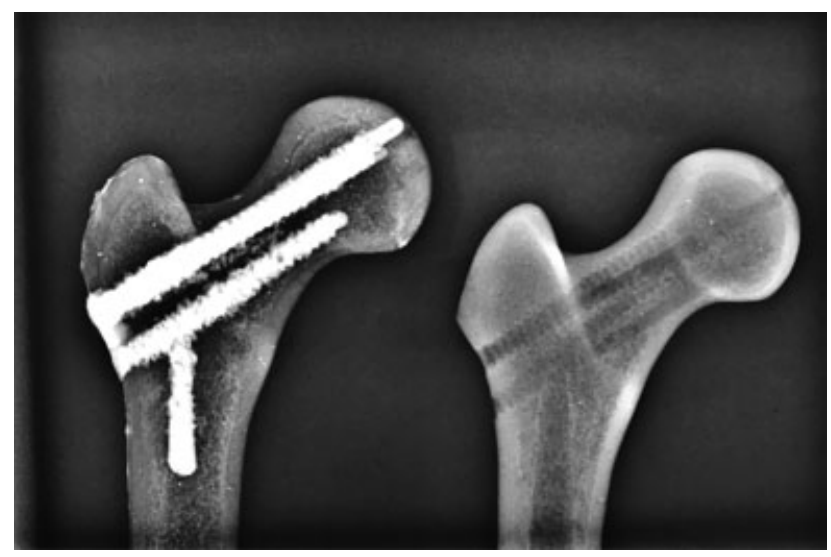

Fig. 1 Radiografia dos fêmures após preenchimento com polimetilmetacrilato (esquerda) e sem reforço (direita).

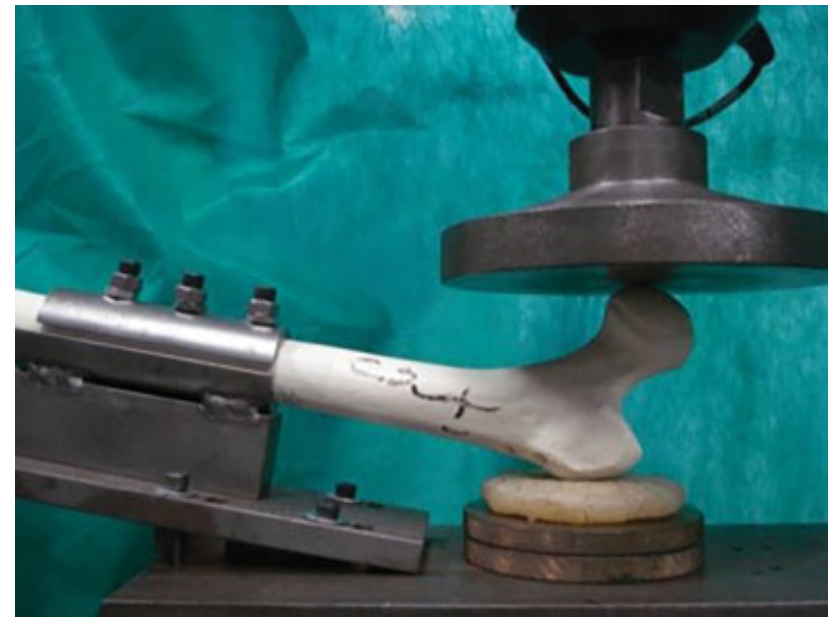

Fig. 2 Osso sintético antes da realização do teste mecânico.

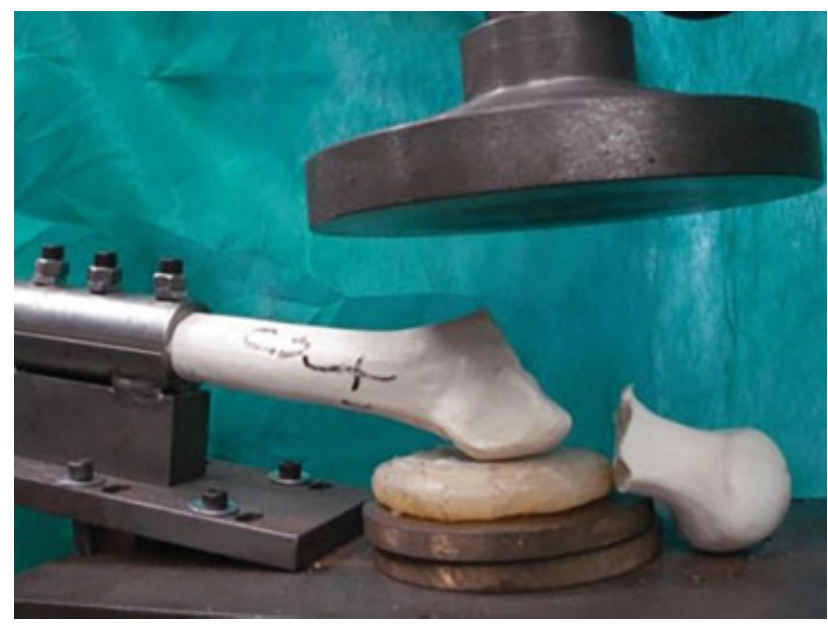

Fig. 3 Osso sintético após a realização do teste mecânico. Observa-se fratura basocervical de espécime.

O fêmur foi fixado ao dispositivo de ensaios deixando $150 \mathrm{~mm}$ do seu comprimento fora do dispositivo de fixação, em direção ao pistão hidráulico, posicionado na base da máquina de ensaios com inclinação de $10^{\circ} \mathrm{com}$ a horizontal e $15^{\circ}$ de rotação interna, aferidos por meio de goniômetro digital, mantendo o trocânter maior apoiado em um disco de silicone de $8 \times 2 \mathrm{~cm}$ de diâmetro. (-Fig. 2) Foi aplicada uma pré-carga de $40 \mathrm{~N}$ e utilizada uma velocidade de $2 \mathrm{~mm} / \mathrm{s}$ de deslocamento do pistão direcionando na cabeça do fêmur até a fratura (-Fig. 3), obtendo-se os valores de carga máxima e carga de escoamento em Newtons (N); energia até o escoamento, energia até a fratura em Joules (J); e rigidez em Newtons por milímetro $(\mathrm{N} / \mathrm{mm})$.

Os resultados foram obtidos por meio de uma análise inferencial, composta pela análise de variância (ANOVA, na sigla em inglês) para um fator juntamente com o teste de comparações múltiplas de Tukey, com o objetivo de verificar se existe diferença significativa na carga máxima e energia até a fratura entre os grupos GC, GTS, GTC. O critério de determinação de significância foi o nível de 5\%. A análise estatística foi processada pelo software estatístico SPSS versão 20.0 (IBM Corp., Armonk, NY, EUA). 


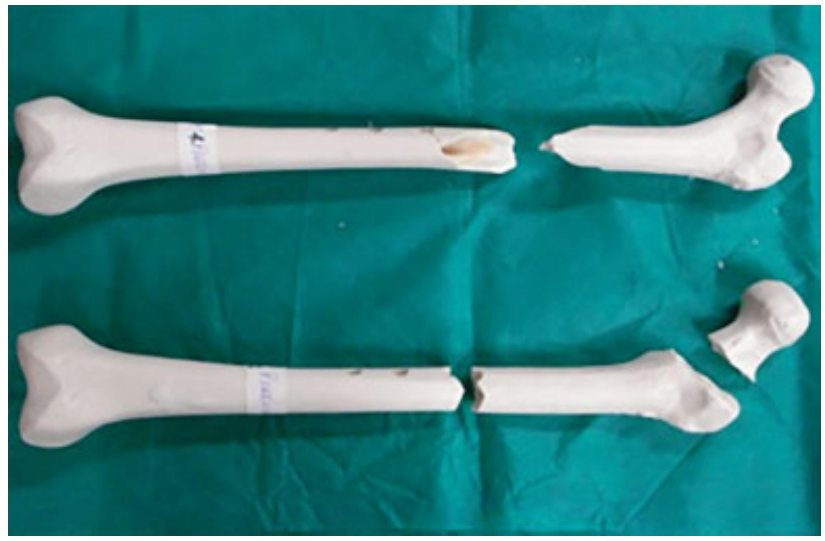

Fig. 4 Espécimes sintéticos com fratura do colo e da diáfise (superior) e da diáfise (inferior) após teste mecânico.

\section{Resultados}

Todos os corpos de prova do GTS e GC apresentaram fratura baso-cervical do colo femoral. No GTC, 3 corpos de prova apresentaram fratura baso-cervical do colo femoral, enquanto que 2 deles apresentaram fratura na parte próxima ao ponto de fixação no dispositivo (região diafisária do fêmur), sendo um deles associado a fratura do colo femoral (-Fig. 4).

A valor médio de PMMA utilizado para preenchimento dos 3 pertuitos dos PCs em cada modelo no grupo GTC foi de $8.2 \mathrm{ml}$.

Os parâmetros analisados nos grupos GC, GTS e GTC apresentaram as seguintes médias respectivamente: carga de escoamento em N (697; 665; 999), energia até escoamento em J ( 2,$8 ; 2,4 ; 3,8)$, rigidez em N/mm (90; 93; 130), carga máxima em $\mathrm{N}(935 ; 886 ; 1565)$ e energia até a fratura em J $(7,1 ; 6,6 ; 16,2)$ (-Figs. 5 e $\mathbf{6})$.

As - Tabelas 1 e $\mathbf{2}>$, fornecem a descritiva dos parâmetros de escoamento/rigidez e carga máxima/energia para fratura, respectivamente, segundo o grupo de preenchimento (GC, GTS e GTC) e o correspondente nível descritivo (valor-p) da

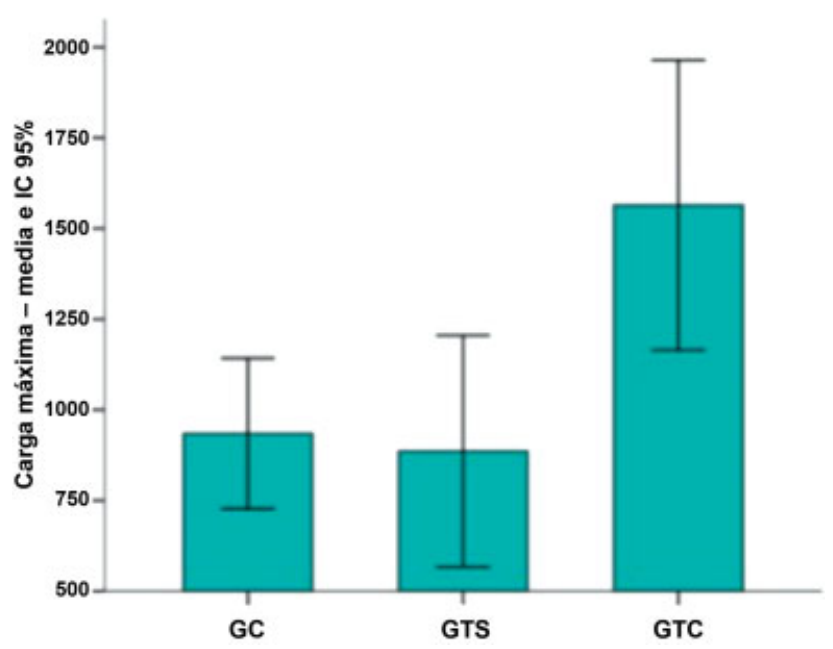

Fig. 5 Gráfico da média de carga máxima e intervalo de confiança de 95\% entre os grupos estudados.

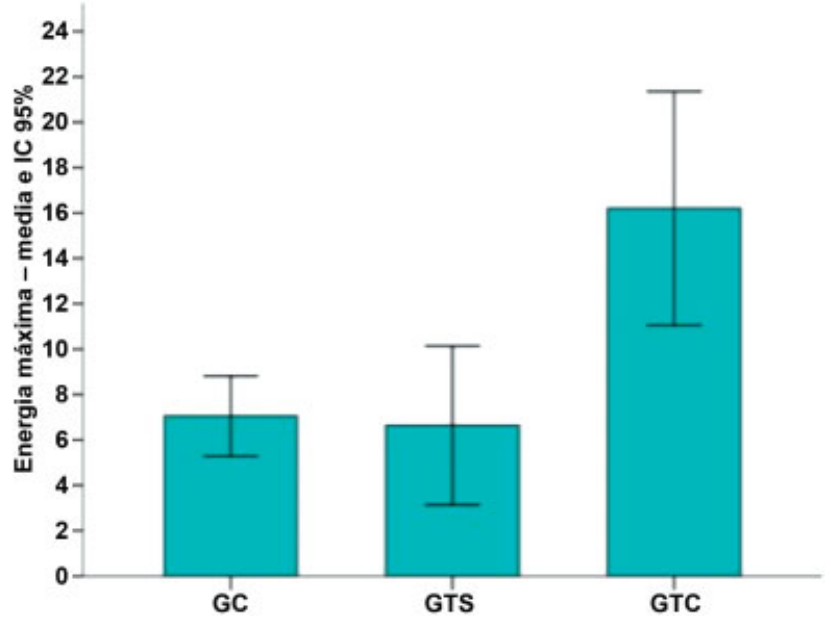

Fig. 6 Gráfico da médica de energia até a fratura e intervalo de confiança de $95 \%$ entre os grupos estudados.

análise de variância (ANOVA) para um fator. O teste de comparações múltiplas de Tukey foi aplicado para identificar os grupos que diferem significativamente entre si, ao nível de $5 \%$, (coluna de $\neq$ significativas da tabela).

Observou-se que o grupo GTC, segundo a ANOVA para um fator, apresentou diferença significativa em relação aos grupos GC e GTS em todos os parâmetros: carga de escoamento $(p=0,029)$, energia até o escoamento $(p=0,044)$, rigidez ( $p=0,035)$, carga máxima $(p=0,001)$ e energia até a fratura $(p=0,0001)$. Quando aplicamos o teste de comparações múltiplas de Tukey, ao nível de 5\%, identificou-se, também, que o grupo GTC apresentou valores significantemente maiores para os mesmos parâmetros (-Tabelas $1 \mathbf{e - 2}$ )

Quando comparamos os grupos GC e GTS, observamos que não há diferença estatística quanto aos parâmetros de carga de escoamento, energia até o escoamento, rigidez, carga máxima e energia até a fratura, segundo a ANOVA para um fator e o teste de comparações múltiplas de Tukey.

\section{Discussão}

As fraturas do fêmur proximal, em especial as do colo femoral, têm em seu tratamento, grande diversidade de métodos e técnicas, destacando-se a fixação com PCs devido a facilitação durante o ato cirúrgico. A configuração em triângulo invertido é objeto de estudo de diversos autores e demonstra ser a opção mecanicamente mais estável., Dessa forma objetivamos utilizar essa conformação por ser mais difundida em nosso meio.

A retirada de implantes do fêmur proximal se mostrou fator de risco para fraturas devido a fragilidade óssea no pertuito do implante retirado. ${ }^{7,8}$ Por essa razão, a retirada do material do fêmur proximal deve ser reservada a pacientes selecionados, principalmente em caso de fragilidade óssea. ${ }^{2,5}$ Com isso, é importante a descrição de estudos que demonstram o comportamento mecânico dessa região após a retirada da síntese.

O uso de modelos sintéticos foi determinado para garantir propriedades biomecânicas comparáveis entre os grupos e 
Tabela 1 Comparativo entre as variáveis carga de escoamento; deslocamento até o escoamento; energia até o escoamento e rigidez quando aplicados os testes estatísticos

\begin{tabular}{|c|c|c|c|c|c|c|c|}
\hline Variável & $n$ & Média & IC 95\% p/ média & Mínimo & Máximo & Valor-p $p^{a}$ & $\neq$ significativa $^{b}$ \\
\hline \multicolumn{8}{|c|}{ Carga de escoamento (N) } \\
\hline GC & 10 & 697 & $559-835$ & 316 & 1.010 & & $\mathrm{Co} \neq \mathrm{cC}$ \\
\hline GTS & 5 & 665 & $480-851$ & 376 & 860 & 0,029 & $\mathrm{sC} \neq \mathrm{cC}$ \\
\hline GTC & 5 & 999 & $865-1133$ & 813 & 1.215 & & \\
\hline \multicolumn{8}{|c|}{ Deslocamento até o escoamento $(\mathrm{mm})$} \\
\hline GC & 10 & 7,7 & $7,0-8,4$ & 5,3 & 9,5 & & \\
\hline GTS & 5 & 7,2 & $6,5-7,8$ & 6,1 & 8,1 & 0,55 & \\
\hline GTC & 5 & 7,7 & $7,1-84$ & 6,7 & 8,4 & & \\
\hline \multicolumn{8}{|c|}{ Energia até o escoamento (J) } \\
\hline GC & 10 & 2,8 & $2,2-3,4$ & 0,9 & 3,8 & & \\
\hline GTS & 5 & 2,4 & $1,6-3,2$ & 1,1 & 3,3 & 0,044 & $\mathrm{sC} \neq \mathrm{cC}$ \\
\hline GTC & 5 & 3,8 & $3,3-4,4$ & 3,1 & 4,5 & & \\
\hline \multicolumn{8}{|c|}{ Rigidez (N/mm) } \\
\hline GC & 10 & 90 & $73-107$ & 60 & 138 & & \\
\hline GTS & 5 & 93 & $69-116$ & 62 & 123 & 0,035 & $\mathrm{Co} \neq \mathrm{cC}$ \\
\hline GTC & 5 & 130 & $108-152$ & 103 & 166 & & \\
\hline
\end{tabular}

Abreviações: GC, grupo controle; GTC, grupo controle com esforço; GTS, grupo controle sem esforço; IC 95\%, intervalo de confiança de $95 \%$. ${ }^{a}$ ANOVA para um fator.

bDiferenças significativas, ao nível de 5\%, segundo o teste de comparações múltiplas de Tukey.

Tabela 2 Comparativo entre as varíaveis carga máxima e energia até a fratura quando aplicados os testes estatísticos

\begin{tabular}{|c|c|c|c|c|c|c|c|}
\hline Variável & $n$ & Média & IC 95\% p/ média & Mínimo & Máximo & Valor- $p^{a}$ & $\neq$ significativa $^{b}$ \\
\hline & \multicolumn{7}{|c|}{ Carga máxima (N) } \\
\hline GC & 10 & 935 & $755-1115$ & 555 & 1.399 & 0,001 & $\mathrm{Co} \neq \mathrm{cC}$ \\
\hline GTS & 5 & 886 & $661-1111$ & 541 & 1.154 & & $\mathrm{sC} \neq \mathrm{cC}$ \\
\hline \multirow[t]{2}{*}{ GTC } & 5 & 1.565 & $1282-1847$ & 1.295 & 2.118 & & \\
\hline & \multicolumn{7}{|c|}{ Energia até fratura (J) } \\
\hline GC & 10 & 7,1 & $5,5-8,6$ & 4,4 & 10,4 & $<0,0001$ & $\mathrm{Co} \neq \mathrm{cC}$ \\
\hline GTS & 5 & 6,6 & $4,2-9,1$ & 3,6 & 10,4 & - & $\mathrm{sC} \neq \mathrm{cC}$ \\
\hline GTC & 5 & 16,2 & $12,6-19,8$ & 11,0 & 22,0 & - & \\
\hline
\end{tabular}

Abreviações: GC, grupo controle; GTC, grupo controle com esforço, GTS, grupo controle sem esforço, IC 95\%, intervalo de confiança de $95 \%$.

eliminar variáveis inerentes aos ossos humanos (densidade óssea, comprimento e diâmetro) que tornariam a avaliação metodológica difícil, além de tornar a execução deste produto quase inviável, pela dificuldade de acesso e pelas leis vigentes em nosso país. $^{9}$

Apesar de os valores absolutos não serem comparáveis aos dos estudos apresentados em ensaios experimentais com ossos de cadáveres, fato esse determinado pela diferença estrutural e biomecânica desses, no caso dos ossos sintéticos, notou-se compatibilidade de resultados quando se observa o incremento de força ao uso de reforço do fêmur proximal com PMMA. ${ }^{10-14}$

O uso de reforço ósseo de PMMA após a retirada de implantes já apresenta resultados experimentais. Há preocupação com o volume usado devido à reação térmica local. ${ }^{9,15} \mathrm{O}$ volume por nós utilizados se aproxima a outros estudos que demonstram pouca variação térmica local. ${ }^{10,14}$

Quando observamos um trabalho similar, no qual o autor usa a mesma metodologia e o mesmo modelo sintético, porém utilizando uma HFP, os autores descrevem resultados estatisticamente significativos ${ }^{15}$; encontramos em nosso estudo, resultado muito intrigante, pois, na HFP, o preenchimento foi realizado em apenas um local específico: túnel do parafuso deslizante de 10,5 mm com um volume de PMMA de $9 \mathrm{ml}$. Notamos que a principal diferença foi a tensão proporcionada pelo reforço com PMMA, pois em nosso estudo tivemos fratura diafisária em dois modelos. Isso nos leva a crer que o local do reforço é mais importante que a quantidade de PMMA utilizado para a técnica e que a cimentação inadvertida dos pertuitos pode trazer complicações inesperadas. 
Aventamos ainda que a possibilidade das fraturas diafisárias nos referidos corpos de prova possam ter ocorrido pelo aparato de fixação, no entanto, dos 20 ossos ensaiados, todos tiveram o mesmo padrão fraturário com o uso do mesmo aparato de fixação. Tal hipótese foi minimizada e atribuída ao real reforço com uso do PMMA e o posicionamento em rotação interna do corpo de prova, uma vez que a carga era aplicada axialmente na cabeça femoral, o movimento de incremento da rotação interna aumentava naturalmente.

Há de considerar ainda como um possível viés na condição clínica que, com a consolidação, há uma diminuição do comprimento real do eixo longo do colo femoral, o que torna o braço de alavanca menor e pode, eventualmente, aumentar a carga e energia necessárias para uma nova fratura.

\section{Conclusão}

A retirada do material de síntese em triângulo invertido do fêmur proximal não demonstrou gerar maior fragilidade óssea quando comparado ao GC. Já o reforço dos orifícios com PMMA demonstrou um incremento da energia e carga máxima significativamente estatístico para a ocorrência de fratura.

Conflitos de Interesse

Os autores declaram não haver conflitos de interesse.

\section{Referências}

1 Gullberg B, Johnell O, Kanis JA. World-wide projections for hip fracture. Osteoporos Int 1997;7(05):407-413

2 Yang JH, Jung TG, Honnurappa AR, Cha JM, Ham CH, Kim TY, et al. The Analysis of Biomechanical Properties of Proximal Femur after Implant Removal. Appl Bionics Biomech 2016;2016:4987831

3 March LM, Chamberlain AC, Cameron ID, Cumming RG, Brnabic AJ, Finnegan TP, et al; Fractured Neck of Femur Health Outcomes Project Team. How best to fix a broken hip. Med J Aust 1999;170 (10):489-494
4 Tosounidis TH, Castillo R, Kanakaris NK, Giannoudis PV. Common complications in hip fracture surgery: Tips/tricks and solutions to avoid them. Injury 2015;46(Suppl 5):S3-S11

5 Eberle S, Wutte C, Bauer C, von Oldenburg G, Augat P. Should extramedullary fixations for hip fractures be removed after bone union? Clin Biomech (Bristol, Avon) 2011;26(04):410-414

6 Basile R, Pepicelli GR, Takata ET. Osteosynthesis of femoral neck fractures: two or three screws? Rev Bras Ortop 2015;47(02): 165-168

7 Kukla C, Pichl W, Prokesch R, Jacyniak W, Heinze G, Gatterer R, et al. Femoral neck fracture after removal of the standard gamma interlocking nail: a cadaveric study to determine factors influencing the biomechanical properties of the proximal femur.J Biomech 2001;34 (12):1519-1526

8 Mahaisavariya B, Sitthiseripratip K, Suwanprateeb J. Finite element study of the proximal femur with retained trochanteric gamma nail and after removal of nail. Injury 2006;37(08):778-785

9 Heini PF, Franz T, Fankhauser C, Gasser B, Ganz R. Femoroplastyaugmentation of mechanical properties in the osteoporotic proximal femur: a biomechanical investigation of PMMA reinforcement in cadaver bones. Clin Biomech (Bristol, Avon) 2004;19(05): 506-512

10 Fliri L, Sermon A, Wähnert D, Schmoelz W, Blauth M, Windolf M. Limited V-shaped cement augmentation of the proximal femur to prevent secondary hip fractures. J Biomater Appl 2013;28(01): 136-143

11 Basafa E, Murphy RJ, Otake Y, Kutzer MD, Belkoff SM, Mears SC, et al. Subject-specific planning of femoroplasty: an experimental verification study. J Biomech 2015;48(01):59-64

12 Cristofolini L, Viceconti M, Cappello A, Toni A. Mechanical validation of whole bone composite femur models. J Biomech 1996;29 (04):525-535

13 Strauss EJ, Pahk B, Kummer FJ, Egol K. Calcium phosphate cement augmentation of the femoral neck defect created after dynamic hip screw removal. J Orthop Trauma 2007;21(05):295-300

14 Beckmann J, Ferguson SJ, Gebauer M, Luering C, Gasser B, Heini P. Femoroplasty-augmentation of the proximal femur with a composite bone cement-feasibility, biomechanical properties and osteosynthesis potential. Med Eng Phys 2007;29(07):755-764

15 Paiva LM, Macedo Neto SL, Souto DR, Ferreira GN, Costa HI, Freitas A. Static bending test after proximal femoral nail (PFN) removal - in vitro analysis. Rev Bras Ortop 2017;52(Suppl 1):52-56 